\title{
Analysis of a One-Dimensional Continuous Delay-Tolerant Network Model
}

\author{
D. Cheliotis*, I. Kontoyiannis ${ }^{\dagger \ddagger}$, M. Loulakis $\$$, and S. Toumpis ${ }^{\ddagger}$ \\ * Department of Mathematics, University of Athens, Greece \\ ${ }^{\dagger}$ Department of Engineering, University of Cambridge, UK

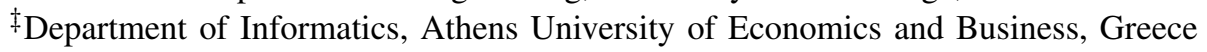 \\ ${ }^{\S}$ School of Applied Mathematical and Physical Sciences, National Technical University of Athens, Greece \\ IInstitute of Applied and Computational Mathematics, Foundation for Research and Technology - Hellas, Greece
}

\begin{abstract}
The packet speed and transmission cost are examined, for a single packet traveling along a simple onedimensional, continuous-time network, using a combination of wireless transmissions and physical transports. We assume that the network consists of two nodes moving at constant speed on a circle, and changing their direction of travel after independent exponential times. The packet wishes to travel in the clockwise direction as fast and as far as possible. It travels either by being physically transported on a node's buffer, or by being wirelessly transmitted to the other node when the two are in the same location. We derive exact, explicit expressions for the long-term average packet speed (in the clockwise direction), and also for the average wireless transmission cost. These results can be viewed as initial steps towards the development of analogous exact expressions for the speed and cost, in more realistic, twodimensional wireless delay-tolerant network models.

Index Terms-Delay, mobile network, one-dimensional network, wireless delay-tolerant network, Markov process, generator, harmonic function
\end{abstract}

\section{INTRODUCTION}

Numerous mobile wireless networks have recently emerged in which packets travel towards their destinations using wireless transmissions as well as physical transport on the buffers of mobile nodes. Examples include space [1], vehicular [2], sensor [13], and pocket switched networks [10]. As the physical transport of data is a relatively slow process, all these networks fall in the wider class of delay-tolerant networks (DTNs) [16].

Models for such networks have been studied and analyzed in a number of earlier works. Notably, the seminal work [9] presented an analysis of the performance of a routing protocol under which each data source gives packets intended for its destination to all the nodes it meets, but each of these transmits the packets only to the destination, whenever the two meet; the protocol was shown to achieve a pernode throughput that did not diminish with the number of nodes, under general assumptions on the mobility model. Similar performance was exhibited even when the nodes are restricted to move along one-dimensional trajectories, on the great circles of a sphere [7].

More recently, the authors of [11] studied the information propagation speed, i.e., the speed with which a single packet propagates in a mobile wireless network, when it is broadcast by any node that receives it to all nodes it subsequently meets.
In that setting, the authors developed bounds on the propagation speed for a variety of mobility models. These results were established using Laplace transforms of the density of spacetime trajectories, and they were validated through simulation experiments.

In [14], the authors considered a network model where nodes move according to Brownian motion, and two nodes can communicate directly with each other whenever they are within a distance $r$ from each other. Here, the authors investigated the statistics of three important quantities: The time until some specified target comes in contact with any of the nodes in the network, the time until all locations within a bounded set come in contact with any of the nodes in the network, and the time until a node becomes a member of an unbounded component of the network graph.

In contrast to the models described above, a lower-level view of the network was adopted in [5], [4], [12], focusing on a single packet that is not replicated as it travels towards a destination located at an infinite distance away. Here, it was assumed that the packet travels using both wireless transmissions and physical transports on the buffers of nodes, according to a general routing rule. The average speed with which the packet approaches its destination was computed, as was the average rate with which transmission cost accumulates; due to the complexity of the problem, judicious approximations were made in these computations.

One-dimensional mobile DTN models, where all nodes are constrained to move along a given line, have also been studied. This type of model may at first appear overly restrictive, however, it is suitable for an important class of DTNs, namely, vehicular networks (VANETS) comprised of cars moving in either single- or multi-lane highways. For example, in [2] the authors studied a bidirectional model with two lanes of traffic moving in opposite directions, and they discovered that a phase transition occurs: If the vehicle densities are above a certain threshold, then data propagate dramatically faster than the vehicle speed, whereas below that threshold data propagate with a speed on average equal to the vehicle speed. Similar settings were investigated in [3], [17].

A common theme of all the works mentioned so far is that, due to the mathematical complexity of the models considered, some form of compromise was always made in their analysis. 
In some cases approximations were introduced (e.g., [5], [4], [12]), in some other cases the relevant results were derived in the asymptotic scenario where the number of nodes grows to infinity (e.g., [9], [7]), or bounds were derived instead of exact expressions (e.g., [11]). Thus motivated, we recently studied a mobile DTN model for which exact, closed-form results could be obtained [6]. The price incurred for such precise results was that attention was restricted to a very simple model: A discrete-time, discrete-space network comprised of only two nodes and a single packet.

In that model the two nodes are assumed to move on a discrete circle of $N$ locations, in one of two possible directions (clockwise (CW) and counter-clockwise (CCW)), and to change their direction of travel in each time slot with probability $\epsilon$. The packet wishes to travel in the CW direction, and it either stays with its current holder, or it gets transmitted to the other node if the two nodes are collocated, its current holder is traveling in the CCW direction, and the other node is traveling in the $\mathrm{CW}$ direction. Closed-form expressions were derived in [6] for the packet speed (i.e., the average net number of $\mathrm{CW}$ steps the packet takes per time slot) and the transmission cost (i.e., the long-term percentage of time slots during which the packet gets transmitted to the other node).

Building on this earlier work [6], here we consider a continuous one-dimensional model, again with two nodes and a single packet. The nodes move in continuous time, with constant speed $v$ in either the $\mathrm{CW}$ or the $\mathrm{CCW}$ direction, on the circumference of a circle of length $\ell$. The nodes change their travel directions, independently of each other, at the event times of a homogeneous Poisson process. The packet remains at the buffer of one of the nodes, until the first instance of the following event, in which case the packet is transmitted to the other node: The two nodes meet, its current holder is moving in the $\mathrm{CCW}$ direction, and the other node is moving in the $\mathrm{CW}$ direction.

The present results are natural continuous analogs of the corresponding results for the discrete-time/discrete-space model of [6]. Nevertheless, it is perhaps somewhat surprising that the probabilistic tools needed for the present analysis are more recent, and of a quite different nature.

In Section II we describe the underlying stochastic model in detail, Section III contains the statements of our two main results, namely the expressions for the long-term average packet speed and transmission cost, and in Sections IV and V we give their proofs.

\section{MODEL}

Let $\mathbb{T}=\mathbb{R} / \ell \mathbb{Z}$ be the one-dimensional circle of length $\ell$. We place two independent walkers on $\mathbb{T}$ and denote their positions at time $t \geq 0$ by $X_{t}=\left(X_{t}(1), X_{t}(2)\right)$. With each walker, $i=1,2$, we associate a velocity at time $t$, with magnitude $v$ and random direction $S_{t}(i)$, where $S_{t}(i)$ is either $=+1(\mathrm{CW}$ motion) or -1 (CCW motion). The initial positions $X_{0} \in \mathbb{T}^{2}$ and directions $S_{0} \in\{-1,+1\}^{2}$ are arbitrary. The evolution of the system can be described as follows. Walker $i \in\{1,2\}$ moves along the circle at constant velocity $S(i) v$, and flips its direction after an exponentially distributed waiting time with mean $1 / r$, independently of the other walker, for some fixed $r>0$.

A single packet is to be forwarded $\mathrm{CW}$ on $\mathbb{T}$ carried by either of the walkers. It only switches from one walker to the other when the two collocated, and it does so, if the current carrier of the packet is moving $\mathrm{CCW}$ while the other walker is moving $\mathrm{CW}$. To track the packet, we define an index process $\left\{I_{t} ; t \geq 0\right\}$ evolving on $\{1,2\}$, with $I_{t}$ denoting the index of the walker carrying the packet at time $t$.

Strictly speaking, $\boldsymbol{\Phi}=\left\{\Phi_{t}=\left(X_{t}, S_{t}, I_{t}\right) ; t \geq 0\right\}$ is a strong Markov process on the state space $\Sigma$ consisting of all configurations $\phi \in \mathbb{T}^{2} \times\{-1,+1\}^{2} \times\{1,2\}$ of the form,

$$
\phi=\left(x_{1}, x_{2}, s_{1}, s_{2}, i\right) \in \mathbb{T}^{2} \times\{-1,+1\}^{2} \times\{1,2\},
$$

where we identify every state of the form $(x, x, s,-s, i)$ with the corresponding state $\left(x, x, s,-s, i^{\prime}\right)$, where, for an index $i \in\{1,2\}$, we denote the complementary index by $i^{\prime}$, i.e., $i^{\prime}=3-i$. We assume that the transitions between directions and between successive values of the index process $\left\{I_{t}\right\}$ are such that the sample paths of $\boldsymbol{\Phi}$ are right-continuous. Since $\Sigma$ is a compact set, this makes $\boldsymbol{\Phi}$ a non-explosive, Borel right process [15], [8].

The dynamics of $\boldsymbol{\Phi}$ are described by its infinitesimal generator, $L$. The domain $\mathcal{D}(L)$ of $L$ consists of functions $f: \Sigma \rightarrow \mathbb{R}$ that are continuously differentiable in their spatial variables for every $s \in\{-1,+1\}^{2}$ and $i \in\{1,2\}$. To describe the action of $L$ explicitly, we first introduce some notation. For $\phi=(x, s, i) \in \Sigma$, let $\sigma^{k} \phi=\left(x, \sigma^{k} s, i\right)$, where $\sigma^{k} s$ is obtained from $s$ by changing the sign of its $k$-th coordinate. Then,

$$
L f(\phi)=\sum_{k=1}^{2} v s(k) \frac{\partial f}{\partial x_{k}}(\phi)+r\left(f\left(\sigma^{k} \phi\right)-f(\phi)\right) .
$$

The first term in the sum on the right-hand side of (1) corresponds to the motion of walker $k$ at constant velocity $v s(k)$, while the second one corresponds to the change of direction at rate $r$.

The following proposition, stated without proof, describes some of the main ergodicity properties of $\boldsymbol{\Phi}$. Throughout, for any measure $\mu$ and function $f$, we write $\mu(f)$ for the integral $\int f d \mu$, whenever it exists.

\section{Proposition 1:}

(i) $\boldsymbol{\Phi}=\left\{\Phi_{t}=\left(X_{t}, D_{t}, I_{t}\right) ; t \geq 0\right\}$ is $\psi$-irreducible and aperiodic on $\Sigma$, with respect to $\psi:=\mathcal{L}^{2} \times \kappa^{2} \times \kappa$, where $\mathcal{L}$ and $\kappa$ denote the Lebesgue and counting measure, respectively.

(ii) $\boldsymbol{\Phi}$ is ergodic, with a unique invariant probability measure $\pi$.

(iii) The following ergodic theorem holds for $\boldsymbol{\Phi}$ : For any bounded (measurable) function $f: \Sigma \rightarrow \mathbb{R}$ and any initial state $\Phi_{0}=\phi \in \Sigma$,

$$
\lim _{t \rightarrow \infty} \frac{1}{t} \int_{0}^{t} f\left(\Phi_{s}\right) d s=\pi(f), \quad \text { a.s. }
$$




\section{RESULTS}

Theorem 1: (packet speed) For any initial state $\Phi_{0}=\phi \in \Sigma$, the long-term average packet speed is given by:

$$
s:=\lim _{t \rightarrow \infty} \frac{1}{t} \int_{0}^{t} v S_{t}\left(I_{t}\right) d t=v \pi(S(I))=\frac{v^{2}}{2 v+r \ell}, \quad \text { a.s. }
$$

For each $t>0$, let $M_{t}$ denote the (random) number of times the packet switches nodes, up to time $t$.

Theorem 2: (transmission cost) For any initial state $\Phi_{0}=$ $\phi \in \Sigma$, the long-term average cost of packet transmissions is given by:

$$
c:=\lim _{t \rightarrow \infty} \frac{M_{t}}{t}=\frac{r v}{2 v+r \ell}, \quad \text { a.s. }
$$

\section{Remarks.}

1) As in the discrete case treated in [6], there is a simple relationship between the speed and the cost: $c=s r / v$. Therefore, on average, the packet gains a CW distance $v / r$ by every jump. This is the average distance traveled by a walker before flipping direction.

2) In the natural discrete-to-continuous limit, the average speed and transmission cost in the discrete case [6] become exactly the expressions in their continuous-time counterparts given in Theorems 1 and 2: Taking $v=1$, in the limit $\epsilon \rightarrow 0, N \rightarrow \infty$, so that $\epsilon N=r \ell$, we recover (2) and (3) precisely.

\section{Proof OF THEOREM 1}

We begin with some simple notation. Let,

$$
\begin{aligned}
D & =\{(z, z): z \in \mathbb{T}\} \subset \mathbb{T}^{2} \\
F & =\left\{\left(z_{1}, z_{2}, s_{1}, s_{2}, i\right) \in \Sigma: z_{1}=z_{2}, s_{1} s_{2}=-1\right\} \subset \Sigma
\end{aligned}
$$

and note that, by the definition of $\Sigma$, for any $\left(z_{1}, z_{2}, s_{1}, s_{2}, i\right) \in$ $F$, we always have $s_{i}=+1$.

The limit in the statement of the theorem exist a.s. by Proposition 1; in order to compute its actual value, we first define the stopping time,

$$
T=\inf \left\{t>0: \Phi_{t} \in F\right\} .
$$

Let $\nu$ denote the uniform probability measure on $F$, i.e., with $s^{*}=(+1,-1)$, let,

$$
\nu=\overline{\mathcal{L}} \times \frac{\delta_{\left(s^{*}, 1\right)}+\delta_{\left(-s^{*}, 2\right)}}{2},
$$

where $\overline{\mathcal{L}}$ is the normalized Lebesgue measure on $D$. Then, $T$ is a regeneration time for $\nu$, in the sense that, if $\Phi_{0} \sim \nu$, then $\Phi_{T} \sim \nu$, as well. We go on to compute $\pi(S(I))$ using the following natural continuous-time generalization of Kac's formula.

Lemma 1: If $T$ is a regeneration stopping time for $\nu$ with $\mathbb{E}_{\nu}(T)<\infty$, then for any bounded measurable function $f$ : $\Sigma \rightarrow \mathbb{R}$ we have,

$$
\mathbb{E}_{\nu}\left(\int_{0}^{T} f\left(\Phi_{s}\right) d s\right)=\mathbb{E}_{\nu}(T) \pi(f)
$$

Proof. If $\mathbb{P}_{\nu}(T=0)=1$, the claim is trivial. Suppose now $0<\mathbb{E}_{\nu}(T)<\infty$, and consider the following occupation measure $\mu$ on $\Sigma$ : For any measurable $A$,

$$
\mu(A)=\mathbb{E}_{\nu}\left(\int_{0}^{T} \mathbb{I}_{A}\left(\Phi_{s}\right) d s\right) .
$$

Observe that $\mu$ integrates measurable functions on $\Sigma$ as,

$$
\int f d \mu=\mathbb{E}_{\nu}\left(\int_{0}^{T} f\left(\Phi_{s}\right) d s\right) .
$$

Let now $g \in \mathcal{D}(L)$. By an application of the optional stopping theorem for the martingale $\left\{g\left(\Phi_{t}\right)-\int_{0}^{t} L g\left(\Phi_{s}\right) d s\right\}$ at the stopping time $T$, we get

$$
\mathbb{E}_{\nu}\left[g\left(\Phi_{T}\right)\right]-\mathbb{E}_{\nu}\left(\int_{0}^{T} L g\left(\Phi_{s}\right) d s\right)=\mathbb{E}_{\nu}\left[g\left(\Phi_{0}\right)\right]
$$

As $T$ is a regeneration time for $\nu$, we have that, $\mathbb{E}_{\nu}\left[g\left(\Phi_{T}\right)\right]=$ $\mathbb{E}_{\nu}\left[g\left(\Phi_{0}\right)\right]$, and hence,

$$
\int L g d \mu=\mathbb{E}_{\nu}\left(\int_{0}^{T} L g\left(\Phi_{s}\right) d s\right)=0 .
$$

Since this holds for all $g \in \mathcal{D}(L)$, the normalized occupation measure $\tilde{\mu}:=\mu / \mu(\Sigma)=\mu / \mathbb{E}_{\nu}(T)$ is an invariant probability measure under the dynamics of $\Phi$, therefore, by uniqueness, $\tilde{\mu}=\pi$, as required.

Next, we compute the expectation of the regeneration time $T$, conditional on the initial state $\Phi_{0}=\phi$ being in $F$.

Lemma 2: For any initial state $\phi \in F$, we have:

$$
\mathbb{E}_{\phi}(T)=\frac{\ell}{v} .
$$

Proof. For any state $\phi=(x, s, i)=\left(x_{1}, x_{2}, s_{1}, s_{2}, i\right) \in$ $\Sigma$, we write $d(x)=x_{1}-x_{2}(\bmod \ell) \in[0, \ell)$. Consider the function $H: \Sigma \rightarrow \mathbb{R}$, defined, for $\phi \notin F$ as,

$$
\begin{aligned}
H(\phi)= & \left(\frac{\ell-2 d(x)}{4 v}\right)\left(s_{1}-s_{2}\right) \\
& +\frac{1+s_{1} s_{2}}{4 r}+\frac{r d(x)(\ell-d(x))}{2 v^{2}},
\end{aligned}
$$

and for $\phi \in F$ as,

$$
H(\phi)=-\frac{\ell}{2 v} .
$$

It is straightforward to verify that $H$ satisfies,

$$
L H(\phi)=-1
$$

for all $\phi \notin F$, and that $H$ is discontinuous across $F$, in that,

$$
\lim _{d(x) \downarrow 0} H(x, s, i)-\lim _{d(x) \uparrow \ell} H(x, s, i)=\frac{\ell}{2 v}\left(s_{1}-s_{2}\right) .
$$

For $\phi \notin F$, an application of the optional stopping theorem for the martingale $\left\{H\left(\Phi_{t}\right)+t\right\}$ gives,

$$
\mathbb{E}_{\phi}(T)=H(\phi)+\frac{\ell}{2 v}, \forall \phi \notin F .
$$


On the other hand, for $\phi \in F$, the Markov property gives,

$$
\mathbb{E}_{\phi}(T)=\mathbb{E}_{\phi}[T ; T \leq t]+\mathbb{E}_{\phi}\left[\mathbb{E}_{\Phi_{t}}(T) ; T>t\right],
$$

for all $t>0$. Since, as $t \rightarrow 0$, we have $\mathbb{P}_{\phi}(T \leq t) \rightarrow 0$ and $H\left(\Phi_{t}\right) \rightarrow \frac{\ell}{2 v}, \mathbb{P}_{\phi}$-a.s., for all $\phi \in F$, letting $t \rightarrow 0$ in the preceding equation and recalling (5) completes the proof.

Now let $\phi^{*}=\left(0,0, s^{*}, 1\right) \in F$ and note that, for all $x \in D$,

$$
\begin{aligned}
\mathbb{E}_{\left(x, s^{*}, 1\right)}\left[\int_{0}^{T} S_{t}\left(I_{t}\right) d t\right] & =\mathbb{E}_{\left(x,-s^{*}, 2\right)}\left[\int_{0}^{T} S_{t}\left(I_{t}\right) d t\right] \\
& =\mathbb{E}_{\phi^{*}}\left[\int_{0}^{T} S_{t}(1) d t\right] .
\end{aligned}
$$

An application of Lemma 1 with $f\left(\Phi_{t}\right)=S_{t}\left(I_{t}\right)$, combined with Lemma 2, gives,

$$
s=\frac{v^{2}}{\ell} \mathbb{E}_{\nu}\left[\int_{0}^{T} S_{t}\left(I_{t}\right) d t\right]=\frac{v^{2}}{\ell} \mathbb{E}_{\phi^{*}}\left[\int_{0}^{T} S_{t}(1) d t\right] .
$$

Therefore, writing $X_{t}^{*}(i)$ for the total CW displacement distance traveled by walker $i=1,2$, up to time $t \geq 0$, so that $X_{t}^{*}(i)=v \int_{0}^{t} S_{t}(i) d t$, we have,

$$
\begin{aligned}
s= & \frac{v}{\ell} \mathbb{E}_{\phi^{*}}\left[X_{T}^{*}(1)\right]=\frac{v}{2 \ell} \mathbb{E}_{\phi^{*}}\left[X_{T}^{*}(1)+X_{T}^{*}(2)\right] \\
& \quad+\frac{v}{2 \ell} \mathbb{E}_{\phi^{*}}\left[X_{T}^{*}(1)-X_{T}^{*}(2)\right] \\
= & \frac{v}{2 \ell} \mathbb{E}_{\phi^{*}}\left[X_{T}^{*}(1)-X_{T}^{*}(2)\right],
\end{aligned}
$$

where we used the fact that, since the two walkers start in opposite directions, we have $\mathbb{E}_{\phi^{*}}\left[X_{T}^{*}(1)+X_{T}^{*}(2)\right]=0$ by symmetry. There are exactly two scenarios for the first meeting of the two walkers starting from $F$ with $S_{0}\left(I_{0}\right)=1$ : In the first one, they meet with directions that are opposite to the ones they started with. In this case, we necessarily have $X_{T}^{*}\left(I_{0}\right)-$ $X_{T}^{*}\left(I_{0}^{\prime}\right)=0$. We call this event $C$. In the second scenario, corresponding to event $C^{c}$, the directions of the walkers are the same as the ones they started with, which necessarily means that $X_{T}^{*}\left(I_{0}\right)-X_{T}^{*}\left(I_{0}^{\prime}\right)=\ell$. Therefore, the speed $s$ is:

$$
s=\frac{v}{2} \mathbb{P}_{\phi^{*}}\left(C^{c}\right) .
$$

The following lemma completes the proof of Theorem 1.

Lemma 3: For any initial state $\phi \in F$ of the form $\phi=$ $\left(x, s_{1}, s_{2}, 1\right)$, we have:

$$
\mathbb{P}_{\phi}\left(C^{c}\right)=\frac{2 v}{2 v+r \ell}
$$

Proof. Consider the function $V: \Sigma \backslash F \rightarrow \mathbb{R}$, defined, for any state $\phi=\left(x, s_{1}, s_{2}, i\right) \notin F$ as,

$$
V(\phi):=\frac{r d(x)+v\left[1+\left(s_{1}-s_{2}\right) / 2\right]}{r \ell+2 v} .
$$

It is straightforward to verify that $L V(\phi)=0$, for every $\phi \notin$ $F$. An application of the optional stopping theorem for the martingale $\left\{V\left(\Phi_{t}\right)\right\}$ gives,

$$
\mathbb{P}_{\phi}\left(C^{c}\right)=V(\phi)
$$

for all $\phi=\left(x, s_{1}, s_{2}, 1\right)$ with $x \notin D$. The proof is concluded by using the Markov property at time $t>0$ and sending $t \rightarrow 0$, as in Lemma 2.

\section{PRoOF OF THEOREM 2}

Let $T_{n}$ be the time of the $n$-th return of $\Phi$ to $F$, i.e., $T_{0}=0$ and inductively, for all $n \geq 1$,

$$
T_{n+1}=\inf \left\{t>T_{n}: \Phi_{t} \in F\right\} .
$$

The number of excursions around $F$ up to time $t \geq 0$ is then

$$
N_{t}=\max \left\{n \geq 0: T_{n} \leq t\right\} .
$$

We also define the (independent) Bernoulli random variables $\left\{X_{n} ; n \geq 1\right\}$, that take the value 1 exactly when the event $C$ occurs in the $n$-th excursion around $F$. Lemma 3 implies that,

$$
\mathbb{P}\left(X_{n}=1\right)=\frac{r \ell}{2 v+r \ell}, \quad \forall n \geq 2 .
$$

In this notation, the total transmission cost $M_{t}$ up to time $t \geq 0$ is given by,

$$
M_{t}=\sum_{n=1}^{N_{t}} X_{n}
$$

Since, by Lemma $2, N_{t} / t \rightarrow v / \ell$, a.s., as $t \rightarrow \infty$, we have,

$$
c:=\lim _{t \rightarrow \infty} \frac{M_{t}}{t}=\frac{v}{\ell} \times \frac{r \ell}{2 v+r \ell}=\frac{r v}{2 v+r \ell}, \quad \text { a.s. }
$$

as claimed.

\section{ACKNOWLEDGMENT}

This work has received funding from the European Union's Horizon 2020 Research and Innovation programme under grant agreement No. 645220 (Road-, Air- and Water-based Future Internet Experimentation - RAWFIE) through the National and Kapodistrian University of Athens. Administration support was provided by the Research Centre of the Athens University of Economics and Business.

Travel funding was received from AUEB and RC-AUEB under grant EP-2818-01.

\section{REFERENCES}

[1] G. Araniti, N. Bezirgiannidis, E. Birrane, I. Bisio, S. Burleigh, C. Caini, M. Feldmann, M. Marchese, J. Segui, and K. Suzuki. Contact graph routing in DTN space networks: overview, enhancements and performance. IEEE Communications Magazine, 53(3):38-46, 2015.

[2] E. Baccelli, P. Jacquet, B. Mans, and G. Rodolakis. Highway vehicular delay tolerant networks: Information propagation speed properties. IEEE Trans. Inf. Theory, 58(3):1743-1756, March 2012.

[3] E. Baccelli, P. Jacquet, B. Mans, and G. Rodolakis. Multi-lane vehicleto-vehicle networks with time-varying radio ranges: Information propagation speed properties. In Proc. IEEE ISIT, Istanbul, Turkey, July 2013.

[4] R. Cavallari, S. Toumpis, and R. Verdone. Analysis of hybrid geographic/delay-tolerant routing protocols for wireless mobile networks. In Proc. IEEE INFOCOM, Honolulu, HI, Apr. 2018.

[5] R. Cavallari, R. Verdone, and S. Toumpis. Cost/speed analysis of mobile wireless DTNs under random waypoint mobility. In Proc. Wiopt, Tempe, AZ, May 2016.

[6] D. Cheliotis, I. Kontoyiannis, M. Loulakis, and S. Toumpis. Exact speed and transmission cost in a simple one-dimensional wireless delaytolerant network. In Proc. IEEE ISIT, Aachen, Germany, June 2017. 
[7] S. N. Diggavi, M. Grossglauser, and D. N. C. Tse. Even one-dimensional mobility increases the capacity of wireless networks. IEEE Trans. Inf. Theory, 51(11):3947-3954, 2005.

[8] D. Down, S. P. Meyn, and R. L. Tweedie. Exponential and uniform ergodicity of Markov processes. Ann. Probab., 23(4):1671-1691, 1995.

[9] M. Grossglauser and D. N. C. Tse. Mobility increases the capacity of ad-hoc wireless networks. In Proc. IEEE INFOCOM, volume 3, pages 1360-1369, Anchorage, AL, Apr. 2001.

[10] P. Hui, J. Crowcroft, and E. Yoneki. Bubble rap: Social-based forwarding in delay-tolerant networks. IEEE Trans. Mobile Comput., 10(11):1576$1589,2011$.

[11] P. Jacquet, B. Mans, and G. Rodolakis. Information propagation speed in mobile and delay tolerant networks. IEEE Trans. Inf. Theory, 56:50015015, Oct. 2010.
[12] I. Kontoyiannis, S. Toumpis, R. Cavallari, and R. Verdone. On calculating packet speed and cost in a mobile wireless network model. In Proc. IEEE ISIT, Vail, CO, June 2018.

[13] N. P. Pathirana, N. Bulusu, A. V. Savkin, and S. Jha. Node localization using mobile robots in delay-tolerant sensor networks. IEEE Trans. Mobile Comput., 4(3):285-296, 2005.

[14] Y. Peres, A. Sincalir, P. Sousi, and A. Stauffer. Mobile geometric graphs: Detection, coverage, and percolation. Probab. Theory Relat. Fields, 156(1):273-305, 2013.

[15] M. Sharpe. General theory of Markov processes. Academic press, 1988.

[16] A. Vasilakos, Y. Zhang, and T. V. Spyropoulos, editors. Delay Tolerant Networks: Protocols and Applications. CRC Press, 2011.

[17] M. Zarei, A. M. Rahmani, and H. Samini. Connectivity analysis for dynamic movement of vehicular ad hoc networks. Wireless Networks, 23(3):843-858, April 2017. 